\title{
Necesidad de generar proyectos alineados a la política regulatoria del sector transporté, para combatir la tasa de mortalidad promovida por los accidentes de tránsito y que afecta a la economía del Ecuador
}

Need to generate projects aligned to the regulatory policy of the transport sector, to combat the mortality rate promoted by traffic accidents and which affects the economy of Ecuador

Lenin Agustín Chamba Bastidas. ${ }^{1}$, María Del Carmen Moreno Albuja. ${ }^{2}$, Rómulo Agustín Cortez Manzano. ${ }^{3} \&$ Katherin Alejandra Carrera Silva. ${ }^{4}$

DOI: https://doi.org/10.33262/visionariodigital.v3i3.667

\begin{abstract}
Economic science is a social science that has a close relationship with the regulation and the mortality rate generated by land traffic accidents, from the allocation of resources to manage regulatory policy that seeks to minimize the mortality rate to the resources that are destined to invest in tools that allow and enable mobility and transit in Ecuador. Annually about 1.3 million people die due to traffic accident issues according to the World Plan for the Decade of Action for Road Safety 2011 - 2020, while in Ecuador the mortality rate is 16\% in 2013 and $13 \%$ in 2018 per 100,000 thousand inhabitants, although the rate goes down by 2018 , it is important to highlight that the management is not enough to lower the rate or maintain the downward trend. If we relate this problem to GDP, the social and economic costs of accidents and injuries amount to $1 \%$ of GNP in low-income countries, $1.5 \%$ in middle-income countries and $5 \%$ in high-income countries. The work is supported by a brief review of the legal field, the scope of national and international planning and data obtained in the National Traffic Agency of Ecuador, institution that is responsible for managing this information. The objective is to analyze the information gathered by means of the chosen methodology in order to establish a value judgment regarding the problems raised.

Keywords: Regulation, Road safety, Transportation, Mortality.

Resumen

La ciencia economía es una ciencia social que tiene una estrecha relación con la regulación y la tasa de mortalidad generada por los accidentes de tránsito terrestre, desde la asignación de recursos para gestionar la política regulatoria que busca minimizar la tasa de mortalidad hasta los recursos que se destina para invertir en herramientas que permitan y habiliten la movilidad y tránsito en el Ecuador. Anualmente alrededor de 1,3 millones de personas fallecen por temas de
\end{abstract}

\footnotetext{
${ }^{1}$ Escuela Superior Politécnica de Chimborazo, Riobamba, Ecuador, lenin.chamba@espoch.edu.ec

${ }^{2}$ Escuela Superior Politécnica de Chimborazo, Riobamba, Ecuador, Carmen.moreno@espoch.edu.ec

3 Corporación Nacional De Telecomunicaciones, Riobamba, Ecuador, agustin2007.cortez@gmail.com

${ }^{4}$ Escuela Superior Politécnica de Chimborazo, Riobamba, Ecuador, Katherin.carrera@espoch.edu.ec
} 
accidentes de tránsito según el Plan Mundial para el Decenio de Acción para la Seguridad Vial 2011 - 2020, mientras que en el Ecuador la tasa de mortalidad es del $16 \%$ en el año 2013 y $13 \%$ en el año 2018 por cada 100.000 mil habitantes, si bien la tasa baja para el 2018, es importante resaltar que la gestión no es suficiente para bajar la tasa o mantener la tendencia a la baja. Si relacionamos este problema con el PIB, los costos sociales y económicos por accidentes y lesionados, ascienden al 1\% del PNB en los países de ingresos bajos, el 1,5\% en los países medios y el $5 \%$ en los países con ingresos altos. El trabajo está sustentado en una revisión breve del ámbito legal, el ámbito de la planificación nacional e internacional y de los datos obtenidos en la Agencia Nacional de Tránsito del Ecuador, institución que se encarga de administrar dicha información. El objetivo es analizar la información recabada mediante la metodología elegida a efectos de establecer un juicio de valor respecto de la problemática planteada.

Palabras clave: Regulación, Seguridad vial, Transporte, Mortalidad.

\section{Introducción}

La regulación permite establecer el hilo conductor que en un momento determinado facilitará lograr los objetivos planteados para beneficio de la sociedad. La muerte y postración de los individuos generada por los accidentes de tránsito, no es un tema aislado, es cada vez más recurrente y requiere mayor atención e importancia por parte de las autoridades, la academia y del público en general. A nivel mundial, la tasa de mortalidad no disminuye como se desea, pero es más grave aun cuando sabemos que en los países con menos recursos, la problemática es más letal con sus habitantes. El Ecuador no está exento de la problemática, al contrario, se evidencia una consolidación del problema, más aún cuando las causas, generadoras y promotoras, siguen sin recibir una atención ambiciosa para eliminarlas de raíz.

Según el Plan Mundial para el Decenio de Acción para la Seguridad Vial 2011-2020, cada año fallecen alrededor de 1,3 millones de personas y entre 20 y 50 millones más, sufren traumatismos provocados por los accidentes de tránsito y el $90 \%$ de las defunciones se dan en los países de ingresos bajos y medianos, datos que tienden a crecer si las medidas no son efectivas. Organismos como la Organización para la Cooperación y el Desarrollo Económico - OCDE, Naciones Unidas - ONU, Organización Mundial de la Salud OMS, Comisión Económica para América Latina - CEPAL, La Asociación LatinoAmericana de Sistemas Integrados y BRT - SIBRT, Movilidad Urbana Sustentable de América Latina-MUSAL, entre otras importantes instituciones a nivel mundial, regional y local, dejan trabajos con alto nivel de calidad e importancia al servicio de los pueblos que lo necesiten, para mejorar su calidad de vida a través de una excelente gestión del transporte terrestre, con excelente estructura en las diferentes aristas del transporte terrestre.

\section{PROBLEMÁTICA}

Cuando apareció el transporte y especialmente el terrestre, de alguna manera se estaba solucionando un problema, principalmente de movilizarse de un lugar a otro, requería de un tiempo mayor si lo hacía caminando o en algún medio que no sean los que actualmente tenemos, sin embargo esta solución a la movilización tradicional, trajo consigo ciertos problemas dada las circunstancias de su aparición, es decir la infraestructura en principio no estaba adaptada al medio de transporte terrestre, se tuvo que adecuar aquella que ya estaba instalada principalmente en las ciudades capitalinas y a partir de allí no se generó 
una apropiada cultura de movilidad y planificación, dando como resultado ciertos efectos negativos que son de conocimiento público en la actualidad.

Para tener una idea de cómo ha evolucionado en el tiempo la tasa de mortalidad generada por accidentes de tránsito y con la finalidad de tener diferentes perspectivas, se revisará algunos trabajos realizados a partir del 2005, año en el que se publicó el trabajo de Rosemarie Plazar. ${ }^{5}$ y en el que se evidencia un número de muertos a nivel mundial, de 1,2 millones cada año, situación que se convertirá en la tercera causa de muerte y discapacidad en el año 2020 por respuesta insuficiente al fenómeno negativo.

Si relacionamos este problema con el PIB, los costos sociales y económicos por accidentes y lesionados, ascienden al 1\% del PNB en los países de ingresos bajos, el 1,5\% en los países medios y el 5\% en los países con ingresos altos. Según el Plan Mundial para el Decenio de Acción para la Seguridad Vial 2011-2020, cada año fallecen alrededor de 1,3 millones de personas - más de 3.000 defunciones diarias, por temas de accidentes de tránsito, lo más alarmante es que, la mitad de ellos no viajaban en automóvil.

Entre 20 y 50 millones de personas, sufren traumatismos, lo que provoca la discapacidad de las personas a nivel mundial. Según el informe sobre la Situación Mundial de la Seguridad Vial $-2013^{6}$, cada año se producen 1,24 millones de muertes a nivel mundial, causadas por accidentes de tránsito, convirtiéndose así en la primera causa de muerte entre los jóvenes de 15 a 29 años, no así con el resto de las personas comprendidas en otro rango de edad.

La muerte o la invalidez de una persona, causada por accidentes de tránsito deja una secuela indeseable para los familiares, más aún cuando la víctima no fue la causante del accidente. Al igual de fuerte la noticia de la muerte de un familiar, es la noticia de la postración de un familiar causada por un accidente de tránsito, los efectos económicos y sociales afectan también a los individuos que no son familiares incluso que no tienen ningún tipo de parentesco, debido las dificultades y vicisitudes que deben pasar para obtener el sustento diario no solo para alimentarse sino también para sostener a la persona postrada, ya que requiere medicamentos y cuidado hasta lograr su mejora en la salud o en ciertos casos de por vida.

Según el mismo documento antes citado, el $90 \%$ de las defunciones causadas por accidentes de tránsito, se encuentran en países de ingresos bajos y medianos y si no se adopta medidas adecuadas, se convertirá en la quinta causa de muerte, lo que aportaría en cierta medida, a la polarización entre las personas de recursos bajos y altos. El tema macroeconómico relacionado al presente trabajo es de alta importancia, ya que podrían ser recursos que se destinen a educación o salud u otros sectores que por su naturaleza no se los puede minimizar ni evitar, recursos que según el mismo informe ascienden aproximadamente del 1 al 3\% del PNB de cada país.

El problema es bastante grave y de rápido crecimiento, mientras más autos hay en las vías y no están habilitadas las condiciones ni exista conciencia y responsabilidad humana, la tasa de mortalidad cada vez será mayor. Es preocupante saber que hay grandes trabajos relacionados al tema, hay una representativa política regulatoria a nivel mundial y nacional y el problema persiste.

\footnotetext{
${ }^{5}$ La seguridad vial en la región de América Latina y el Caribe. Situación actual y desafíos.

${ }^{6}$ Organización Mundial de la Salud
} 


\section{OBJETIVO}

Analizar la tasa de mortalidad generada por accidentes de tránsito y la necesidad de generar proyectos que, mediante la política regulatoria, mejoren la economía del Ecuador.

\section{MATERIALES Y MÉTODOS}

El trabajo de investigación posee un estudio prospectivo y analítico con un fuerte componente legal, al cual los involucrados directos e indirectos debieron prestar extrema atención a fin de dar cumplimiento cabal y oportuno para evitar acción legal alguna. Se tomó como referencia los datos y la información en general que las instituciones internacionales dedicadas al tema ponen a disposición del público y especialmente de las autoridades competentes, para bajar a cero la tasa de mortalidad causada por los accidentes de tránsito.

Este método deductivo que se utilizó permite tener un panorama general de la problemática como también del planteamiento con el que cada región, país, sector e institución considera o pretende solucionar tan impactante problema. A nivel internacional como nacional, las instituciones poseen información cuantitativa y cualitativa respecto del tema, información que permite ofrecer un análisis que sirva de apoyo en todo el proceso investigativo que se realiza para aportar a la mejor calidad de vida de las personas.

\section{FUNDAMENTACIÓN TEÓRICA}

La fundamentación teórica es muy importante en el trabajo de investigación, sin embargo, no suple lo que está descrito en la Ley vigente, esto significa que, en primera instancia, debemos cumplir lo que ordena la Ley y en forma supletoria o en concordancia, integrar el campo teórico. El transporte como tal, se convierte en un eje transversal en la movilidad humana, en el intercambio de bienes y servicios que la sociedad demanda, y en el comercio a nivel mundial.

\section{Economía}

Es la ciencia social que estudia las elecciones que hacen los individuos, las empresas, los gobiernos y las sociedades, para enfrentar la escasez y los incentivos que influyen en esas elecciones y las concilian. (Michael \& Eduardo, 2016)

\section{Estrategia}

Es el proceso mediante el cual quienes toman decisiones en una organización obtienen, procesan y analizan información pertinente, interna y externa, con el fin de evaluar la situación presente de la empresa, así como su nivel de competitividad con el propósito de anticipar y decidir sobre el direccionamiento de la institución hacia el futuro. (Serna G., 1997)

\section{Plan estratégico}

La importancia de la planeación estratégica radica en "establecer los objetivos que la empresa desea conseguir a largo plazo, y se convierten en la guía para desarrollar los planes de acción de mediano y largo plazo que ayudan a lograr objetivos específicos para la empresa." (Morales C. \& Morales C., 2014)

\section{Transporte}

Se define como el traslado de algún elemento de un lugar a otro, ya sean personas o bienes, incluidos los fluidos. El transporte es una actividad fundamental dentro del desarrollo de la humanidad" (Valles, 2013) 


\section{Transporte Terrestre}

Un servicio público esencial y una actividad económica estratégica del Estado, que consiste en la movilización libre y segura de personas o de bienes de un lugar a otro, haciendo uso del sistema vial nacional, terminales terrestres y centros de transferencia de pasajeros y carga en el territorio ecuatoriano. Su organización es un elemento fundamental contrala informalidad, mejorar la competitividad y lograr el desarrollo productivo, económico y social del país. (Asamblea Nacional Constituyente, 2014)

\section{Proyectos}

Conjunto de actividades que se desarrollan en forma coherente con el propósito de obtener un resultado final como respuesta a una necesidad u oportunidad de negocio, en un tiempo determinado y mediante la utilización de recursos. (Murcia \& Piraquive, 2014) Un proyecto es, ni más ni menos, la búsqueda de una solución inteligente al planteamiento de un problema tendiente a resolver, entre tantos, una necesidad humana. Cualquiera que sea la idea que se pretende implementar, la inversión, la metodología o la tecnología por aplicar, ella conlleva necesariamente la búsqueda de proposiciones coherentes destinadas a resolver las necesidades de la persona humana. (Sapag \& Chain, 2008)

Un proyecto de inversión es una propuesta técnica y económica para resolver un problema de la sociedad utilizando los recursos humanos, materiales y tecnológicos disponibles, mediante un documento escrito que comprende una serie de estudios que permiten al inversionista saber si es viable su realización. (Córdoba, 2011)

\section{Política}

Conjunto de actividades e instrumentos disponibles para intervenir y mejorar determinados aspectos de la economía o de sus apartados, como pueden ser la política monetaria, política social, etc. (Arthur Andersen, S. A., 1999)

\section{Regulación}

Intervención del Estado en el funcionamiento normal de la actividad económica mediante la emisión de normas con el objeto de vigilar o modificar determinadas condiciones, que pueden ir desde la fijación de los precios de determinados productos, pasando por la defensa de la competencia en el mercado, o la protección de la situación de los trabajadores. (Arthur Andersen, S. A., 1999)

\section{Externalidades}

Beneficios o costos sociales que se derivan de la realización de una actividad privada y no son contabilizados en esta. Por ejemplo, la contaminación es un daño que sufre la sociedad, pero no un gasto que el productor privado pague en consonancia. (Arthur Andersen, S. A., 1999)

Ley

En derecho, norma jurídica dictada por el Estado, que ordena, permite o prohíbe algo. En ocasiones, el término se generaliza a reglamentos, decretos, ordenanzas, etc., aunque no sean ley en sentido estricto. (Arthur Andersen, S. A., 1999)

\section{Fundamentación Legal}

En derecho, es fundamental observar la jerarquía de las leyes, ya que permiten identificar la relación, vinculación o si se contraponen entre ellas considerando su jerarquía, razón suficiente para considerar la pirámide de Kelsen ${ }^{7}$ a efectos del desarrollar este ámbito.

\section{Constitución de la República del Ecuador del 2008}

En el Art. 85 de la Constitución se observa que la formulación, ejecución, evaluación y

\footnotetext{
${ }^{7}$ Hans Kelsen, profesional y docente de derecho, tiene a su haber grandes obras al servicio de la comunidad.
} 
control de las políticas públicas y servicios públicos que garanticen los derechos reconocidos por la Constitución, se regularán de acuerdo con tres disposiciones, todas importantes y de utilidad, por lo que se resalta lo siguiente de cada una de ellas: a) Las políticas públicas están orientadas al buen vivir. b) Conciliaran los derechos en conflicto observando la prevalencia del interés general sobre el particular. c) El Estado garantizará la distribución equitativa y solidaria del presupuesto para la ejecución de las políticas públicas.

Es de conocimiento general que la Constitución es la Ley suprema en el Ecuador, por lo tanto, la normativa de los diferentes sectores debe alinearse a lo ordenado en la carta magna y partimos del literal a del párrafo antes descrito Las políticas públicas están orientadas al buen vivir, y nace la pregunta que se entiende por buen vivir, de pronto una tasa baja de mortalidad es parte o no del buen vivir, sus políticas están cumpliendo o es necesario hacer más para bajar a cero la tasa de mortalidad.

El Plan Nacional de Desarrollo del Ecuador, es el hilo conductor para el gobierno central y también para los gobiernos autónomos conforme la concordancia con los siguientes artículos.

Los gobiernos regionales autónomos ${ }^{8}$ tendrán las competencias descritas en el Art. 262, sin perjuicio de las otras que determine la ley que regule el sistema nacional de competencias, si bien, todas son importantes, la que nos interesan conocer y trabajar son las siguientes: a) Planificar el desarrollo regional y formular los correspondientes planes de ordenamiento territorial, de manera articulada con la planificación nacional, provincial, cantonal y parroquial. b) Planificar, regular y controlar el tránsito y el transporte regional y el cantonal en tanto no lo asuman las municipalidades. c) Planificar, construir y mantener el sistema vial de ámbito regional.

Los gobiernos municipales tendrán las siguientes competencias exclusivas descritas en el Art. 264 sin perjuicio de otras que determine la ley: a) Planificar el desarrollo cantonal y formular los correspondientes planes de ordenamiento territorial, de manera articulada con la planificación nacional, regional, provincial y parroquial, con el fin de regular el uso y la ocupación del suelo urbano y rural. b) Planificar, construir y mantener la vialidad urbana. c) Planificar, regular y controlar el tránsito y el transporte público dentro de su territorio cantonal.

Los gobiernos parroquiales rurales ejercerán las siguientes competencias exclusivas descritas en el Art. 267, sin perjuicio de las adicionales que determine la ley: a) Planificar y mantener, en coordinación con los gobiernos provinciales, la vialidad parroquial rural. Las políticas, programas y proyectos públicos, la coordinación de las competencias exclusivas entre el Estado central y los gobiernos autónomos descentralizados y otros, se sujetarán al Plan Nacional de Desarrollo conforme el Art. 280 de la constitución, que para el período 2017 - 2022, se lo conoce como PLAN PARA TODA UNA VIDA.

El capítulo primero del título VI, ordena a partir del artículo 275 de la Constitución del Ecuador que, el Estado planificará el desarrollo del país para garantizar el ejercicio de los derechos, la consecución de los objetivos del régimen de desarrollo y los principios consagrados en la Constitución.

Uno de los objetivos del régimen de desarrollo es mejorar la calidad y esperanza de vida según el numeral 1 del artículo 276 de la Constitución, objetivo que demanda una muy alta responsabilidad por parte del Estado, claro siempre y cuando haya el deseo por parte de las autoridades en cumplir.

\footnotetext{
8 Gobiernos Autónomos Descentralizados
} 
En la Constitución también se menciona que hay sectores denominados estraticos, siendo el Transporte uno de los sectores estratégicos, por lo tanto y según el artículo 313, el Estado se reserva el derecho de administrar, regular, controlar y gestionar este y otros sectores.

Por estar relacionado con el presente trabajo y dada la importancia del contenido, citaremos textualmente el siguiente artículo:

Art. 394.- El Estado garantizará la libertad de transporte terrestre, aéreo, marítimo y fluvial dentro del territorio nacional, sin privilegios de ninguna naturaleza. La promoción del transporte público masivo y la adopción de una política de tarifas diferenciadas de transporte serán prioritarias. El Estado regulará el transporte terrestre, aéreo y acuático y las actividades aeroportuarias y portuarias.

\section{Ley Orgánica de Transporte Terrestre Tránsito y Seguridad Vial.}

Art. 1.- La presente Ley tiene por objeto la organización, planificación, fomento, regulación, modernización y control del Transporte Terrestre, Tránsito y Seguridad Vial, con el fin de proteger a las personas y bienes que se trasladan de un lugar a otro por la red vial del territorio ecuatoriano, y a las personas y lugares expuestos a las contingencias de dicho desplazamiento, contribuyendo al desarrollo socio-económico del país en aras de lograr el bienestar general de los ciudadanos.

Art. 2.- La presente Ley se fundamenta en los siguientes principios generales: el derecho a la vida, al libre tránsito y la movilidad, la formalización del sector, lucha contra la corrupción, mejorar la calidad de vida del ciudadano, preservación del ambiente, desconcentración y descentralización interculturalidad e inclusión a personas con discapacidad.

Uno de los principios es el derecho a la vida, del cual se desprende la incógnita de cómo hacer valer el derecho o que se requiere para evitar las muertes causadas por accidentes de tránsito, así mismo el articulo 3 describe e indica que el Estado garantizará que la prestación del servicio de transporte público se ajuste a los principios de seguridad, eficiencia, responsabilidad, universalidad, accesibilidad, continuidad y calidad, con tarifas socialmente justas.

Art. 4.- Es obligación del Estado garantizar el derecho de las personas a ser educadas y capacitadas en materia de tránsito y seguridad vial, en su propia lengua y ámbito cultural. Para el efecto, el Ministerio del Sector de la Educación en coordinación con la Agencia Nacional de Regulación y Control del Transporte Terrestre, Tránsito y Seguridad Vial, desarrollarán los programas educativos en temas relacionados con la prevención y seguridad vial, principios, disposiciones y normas fundamentales que regulan el tránsito, su señalización considerando la realidad lingüística de las comunidades, pueblos y nacionalidades, el uso de las vías públicas, de los medios de transporte terrestre y dispondrán su implementación obligatoria en todos los establecimientos de educación, públicos y privados del país.

Educar a las personas es incrementar la cultura vial, es disminuir la tasa de accidentes y por ende la tasa de mortalidad, sin embargo, estas no bajan al nivel deseado.

Art. 5.- El Estado, a través de la Agencia Nacional de Regulación y Control del Transporte Terrestre, Tránsito y Seguridad Vial, controlará y exigirá la capacitación integral, permanente, la formación y tecnificación a conductoras y conductores profesionales y no profesionales y el estricto cumplimiento del aseguramiento social.

Art. 13.- Son órganos del transporte terrestre, tránsito y seguridad vial, los siguientes: a) El Ministerio del Sector; b) La Agencia Nacional de Regulación y Control del Transporte 
Terrestre, Tránsito y Seguridad Vial y sus órganos desconcentrados; y, c) Los Gobiernos Autónomos Descentralizados Regionales, Metropolitanos y Municipales y sus órganos desconcentrados.

Son los órganos descritos en el artículo 13, los responsables de gestionar la política pública en beneficio de los usuarios y ciudadanía en general.

Art. 15.- El Ministro del Sector será el responsable de la rectoría general del sistema nacional de transporte terrestre, tránsito y seguridad vial en coordinación con los GADs, expedirá el Plan Nacional de Movilidad y Logística del transporte y supervisará y evaluará su implementación y ejecución.

Art. 16.- La Agencia Nacional de Regulación y Control del Transporte Terrestre, Tránsito y Seguridad Vial, es el ente encargado de la regulación, planificación y control del transporte terrestre, tránsito y seguridad vial en el territorio nacional, en el ámbito de sus competencias, con sujeción a las políticas emanadas del Ministerio del Sector; así como del control del tránsito en las vías de la red estatal-troncales nacionales, en coordinación con los GADS y tendrá su domicilio en el Distrito Metropolitano de Quito.

La Agencia Nacional de Regulación y Control del Transporte Terrestre, Tránsito y Seguridad Vial es una entidad autónoma de derecho público, con personería jurídica, jurisdicción nacional, presupuesto, patrimonio y régimen administrativo y financiero propios.

\section{Clases de servicios de Transporte Terrestre}

Según artículo 51 de la Ley Orgánica de Transporte Tránsito y Seguridad Vial, se establecen las siguientes clases de servicios de transporte terrestre:
a. Público;
b. Comercial;
c. Por cuenta propia; y,
d. Particular.

\section{Código Orgánico de Ordenamiento Autónomo Descentralizado (COOTAD)}

Entre los varios objetivos citados en el artículo 2 del COOTAD, citaremos el literal a y el literal $\mathrm{b}$ únicamente, sin pretender que se entienda que los demás no son importantes.

Art. 2.- Objetivos. - Son objetivos del presente Código:

a) La autonomía política, administrativa y financiera de los gobiernos autónomos descentralizados, en el marco de la unidad del Estado ecuatoriano; b) La profundización del proceso de autonomías y descentralización del Estado, con el fin de promover el desarrollo equitativo, solidario y sustentable del territorio, la integración y participación ciudadana, así como el desarrollo social y económico de la población...

Art. 4.- Fines de los gobiernos autónomos descentralizados. - Dentro de sus respectivas circunscripciones territoriales son fines de los gobiernos autónomos descentralizados:

a) El desarrollo equitativo y solidario mediante el fortalecimiento del proceso de autonomías y descentralización; ... d) La recuperación y conservación de la naturaleza y el mantenimiento de medio ambiente sostenible y sustentable; ... g) El desarrollo planificado participativamente para transformar la realidad y el impulso de la economía popular y solidaria con el propósito de erradicar la pobreza, distribuir equitativamente los recursos y la riqueza, y alcanzar el buen vivir; ... 


\section{Fundamentación de la Planificación Internacional}

\section{Organización para la Cooperación y el Desarrollo Económico - OCDE}

La misión de la organización es promover políticas que mejoren el bienestar económico y social de las personas alrededor del mundo.

El Comité de Política Regulatoria - OCDE realiza las siguientes recomendaciones en el año 2012, tanto para los países miembros cuanto para aquello que no lo son.

1. Comprometerse al más alto nivel político con una política expresa de gobierno completo para la calidad regulatoria. ...

2. Adherirse a los principios de gobierno abierto, considerando la transparencia y la participación en el proceso regulatorio a fin de asegurar que la regulación se encuentre al servicio del interés público y esté informada de las necesidades legítimas de aquellos a quienes concierne y afecta...

3. Establecer mecanismos e instituciones para supervisar activamente los procedimientos y objetivos de la política regulatoria, apoyarla e implementarla, y por consecuencia fomentar la calidad de la regulación.

4. Integrar la Evaluación de Impacto Regulatorio (EIR) a las primeras etapas del proceso de diseño de políticas públicas para formular proyectos regulatorios nuevos. Identificar claramente las metas de política pública, y evaluar si es necesaria la regulación y de qué manera puede ser más efectiva y eficiente para alcanzar dichas metas. Tomar en cuenta los medios diferentes de la regulación y determinar la retribución de los diversos enfoques analizados para identificar el mejor.

5. Revisar de forma sistemática el inventario de regulación significativa con base en metas de política pública claramente definidas, incluida la consideración de costos y beneficios, a fin de asegurar que las regulaciones estén actualizadas, se justifiquen sus costos, sean rentables y consistentes, y cumplan con los objetivos de política pública planteados.

6. Publicar de manera periódica informes acerca del desempeño de la política regulatoria y los programas de reforma, así como de las autoridades públicas encargadas de aplicar las regulaciones....

7. Desarrollar una política congruente que contemple el papel y las funciones de las entidades regulatorias a fin de infundir mayor confianza de que las decisiones en cuestiones regulatorias se toman a partir de criterios objetivos, imparciales y coherentes, sin conflictos de interés, prejuicios ni influencias indebidas.

8. Asegurar la efectividad de los sistemas para revisar la legalidad y la justicia procesal de las regulaciones y decisiones que toman los órganos facultados para emitir sanciones en el ámbito regulatorio....

9. Aplicar, cuando se considere adecuado, la evaluación, la gestión y las estrategias de comunicación de riesgos al diseño y la implementación de las regulaciones para asegurar que éstas estén bien orientadas y sean efectivas. Los reguladores deben evaluar de qué manera tendrán efecto las regulaciones y diseñar estrategias de implementación y aplicación sensibles.

10. Promover, cuando sea adecuado, la coherencia regulatoria a través de mecanismos de coordinación entre los niveles de gobierno supranacional, nacional y subnacional. Identificar los problemas transversales en materia de regulación en todos los órdenes de gobierno, con el objeto de promover la coherencia entre los enfoques regulatorios y evitar la duplicidad o conflicto de regulaciones.

11. Impulsar el desarrollo de capacidades y el desempeño de la gestión regulatoria en los órdenes subnacionales de gobierno. 
12. Tomar en cuenta, al desarrollar medidas regulatorias, todos los estándares y marcos internacionales de cooperación pertinentes en la misma área y, cuando sea adecuado, los probables efectos que tengan en terceros fuera de su jurisdicción. ${ }^{9}$

El Comité de Política Regulatoria supervisará la implementación de la recomendación e informará al Consejo en un período no mayor a tres años, después de su adopción. Tomamos en cuenta este documento que data del año 2012, con la intención de ir revisando el camino transcurrido a la fecha en lo que respecta a la política regulatoria del Ecuador.

Si analizamos cada una de las recomendaciones citadas, es muy evidente que se las debe considerar en la planificación local o de Estado, incluso en los Gobiernos Autónomos Descentralizados, son recomendaciones que encajan muy flexiblemente a nuestra realidad.

\section{Plan Mundial para el Decenio de Acción para Seguridad Vial 2011 - 2020}

El reconocer que existe un error humano, permite desarrollar mejores vehículos, mejores vías, un sistema integrado con más soluciones preventivas. La finalidad y objetivos específicos del plan del Decenio principalmente es reducir las víctimas mortales en accidentes de tránsito a nivel mundial antes del 2020.

Para cumplir con los objetivos y la planificación en general, es necesario cumplir con la ejecución de ciertas actividades, en consideración al marco jurídico respectivo y a los siguientes pilares que se describen.

1. Gestión de la seguridad vial

2. Vías de tránsito y movilidad más seguras

3. Vehículos más seguros

4. Usuarios de vías de tránsito más seguros

5. Respuesta tras los accidentes

La realización de las actividades no puede quedar en su ejecución, necesitan obligatoriamente su medición para comprobar la evolución y tomar decisiones oportunas que permitan cumplir con los objetivos planificados, motivo por el cual, vamos a revisar los indicadores propuestas en el Plan por cada pilar y los indicadores de las actividades internacionales.

Indicadores Básico del pilar 1 - Gestión de seguridad vial

1. número de países que se han adherido a los acuerdos y los convenios y convenciones de las Naciones Unidas sobre seguridad vial;

2. número de nuevos instrumentos jurídicos sobre seguridad vial elaborados (y número de países que participan en ellos);

3. número de países que cuentan con un organismo claramente facultado que dirige la seguridad vial;

4. número de países con una estrategia nacional;

5. número de países con metas relativas a la seguridad vial con plazos definidos;

6. número de países que disponen de sistemas de datos para seguir de cerca los avances registrados en la consecución de las metas de seguridad vial;

7. número de países que recopilan datos anuales sobre los accidentes de tránsito congruentes con las definiciones aceptadas a nivel internacional.

\footnotetext{
${ }^{9}$ Recomendación del Consejo sobre Política y Gobernanza Regulatoria - OCDE
} 
Indicadores Básico del pilar 2 - Vías de tránsito y movilidad más segura

1. número de países cuyas autoridades viales tienen la responsabilidad legal de mejorar la seguridad vial en sus redes;

2. número de países con una asignación definida de gastos para programas dedicados a la seguridad de la infraestructura viaria;

3. número de países con una meta para eliminar las vías de tránsito de alto riesgo antes de 2020;

4. número de países que han adoptado políticas sostenibles de movilidad urbana;

5. número de países con unidades especializadas en seguridad vial de las infraestructuras que siguen de cerca los aspectos de seguridad de la red de carreteras;

6. número de países que cuentan con políticas y prácticas establecidas para realizar auditorías de seguridad sistemáticas y evaluar las repercusiones en la seguridad o las vías de tránsito;

7. número de países que se han adherido a los acuerdos regionales de infraestructura viaria, elaborados bajo los auspicios de las comisiones regionales de las Naciones Unidas, y/o que los han aplicado plenamente;

8. número de nuevos instrumentos regionales de infraestructura viaria elaborados (y número de países que participan en ellos).

Indicadores Básico del pilar 3 - Vehículos más seguros

1. número de países que participan en el Foro Mundial de las Naciones Unidas para la Armonización de Reglamentaciones sobre Vehículos y aplican las normas pertinentes; número de países

2. número de países que promulgan leyes que prohíben la utilización de vehículos sin cinturones de seguridad (en los asientos delanteros y traseros).

Indicadores Básico del pilar 4 - Usuarios de vías de tránsito más seguras

1. número de países que establecen límites de velocidad apropiados según el tipo de carretera (urbana, rural, autopista);

2. número de países que fijan límites de alcoholemia inferiores o iguales a $0,05 \mathrm{~g} / \mathrm{dl}$;

3. número de países que fijan límites de alcoholemia inferiores a 0,05 g/dl para los conductores jóvenes o noveles y comerciales;

4. número de países que cuentan con datos nacionales sobre la proporción de accidentes mortales relacionados con el alcohol;

5. número de países que cuentan con una legislación integral sobre el uso del casco (con inclusión de las normas);

6. número de países que cuentan con datos nacionales sobre las tasas de uso del casco;

7. número de países que cuentan con una legislación integral sobre el cinturón de seguridad;

8. número de países que cuentan con datos nacionales sobre las tasas de uso del cinturón de seguridad (en los asientos delanteros y traseros);

9. número de países que cuentan con una legislación sobre los sistemas de retención para niños;

10. número de países con una política oficial para reglamentar el factor cansancio entre los conductores de vehículos comerciales.

Indicadores Básico del pilar 5 - Respuesta tras los accidentes

1. número de países que exigen sistemas de seguros de responsabilidad civil a todos los 
conductores;

2. número de países que cuentan con un número telefónico nacional de acceso en caso de emergencias;

3. número de países que cuentan con centros específicos de atención traumatológica.

Indicadores básicos de Actividades internacionales

1. número de defunciones por accidentes de tránsito, como indicador compuesto básico para todas las actividades;

2. cantidad de fondos procedentes de la comunidad internacional de donantes (incluidos los organismos de desarrollo y donación, las fundaciones, el sector privado y otros donantes) que se destina a la seguridad vial;

3. cantidad de fondos puestos a disposición a escala regional y subregional en apoyo de la seguridad vial; y

4. número de organizaciones e instituciones regionales o subregionales que determinan metas de seguridad vial para reducir para 2020 el número de víctimas mortales causadas por el tráfico rodado. ${ }^{10}$

\section{Fundamentación de la Planificación Nacional \\ Plan Estratégico de Movilidad 2013 - 2037}

El Plan Estratégico de Movilidad del Ministerio de Transporte y Obras Públicas del Ecuador, tiene un horizonte más amplio, llega hasta el año 2037 con su plan, incluso pasa el horizonte del Plan del Decenio que llega hasta el 2021 y de la Agenda 2030.

Se nota claramente que este plan, es muy ambicioso en el sentido de obtener logros representativos para el bienestar del Estado y de sus habitantes, no obstante, ese bienestar y la ejecución de las actividades como el cumplimiento de los objetivos, se lo ira evaluando periódicamente. Si bien la planificación y los proyectos ayudan a minimizar los riesgos, estos no garantizan que en el camino no se presenten obstáculos que impidan obtener los resultados esperados.

El plan orienta el desarrollo del sistema de transporte de Ecuador hasta el año 2037, se definirá los programas de actuación respecto de infraestructuras, equipamiento, institucional y de gestión.

Este plan es específico para la movilidad en el Ecuador en todas sus alternativas de transporte, dado el alcance del trabajo, vamos a ceñirnos en el transporte terrestre, considerando todos los objetivos del plan a sus diferentes períodos de alcance.

Objetivos del horizonte 2020

1. Ejecutar las actuaciones en curso y previstas en el corto plazo

2. Modernización del marco legal vigente y de la organización institucional, potenciando la rectoría del MTOP como Autoridad Oficial Única en la materia y adecuando los modelos de gestión de las infraestructuras

3. Modernización del marco legal vigente complementario a la actividad del MTOP (concesiones, expropiaciones, etc.)

4. Desarrollo de la normativa técnica específica necesaria para el diseño y construcción de las infraestructuras

5. Diseño de los procedimientos administrativos necesarios para los distintos trámites asociados al planeamiento, diseño y construcción de las infraestructuras, y la gestión

${ }^{10}$ Plan Mundial para el Decenio de Acción para Seguridad Vial 2011 - 2020 
y fiscalización de las concesiones, incluida la elaboración de los términos de referencia para las licitaciones.

6. Adecuación y centralización en el MTOP de las actividades de planificación del Sistema de Transportes

7. Capacitación de todos los cuerpos del Ministerio dedicado a labores de dirección, de planificación y de gestión, así como capacitación del cuerpo técnico dedicado a las labores de conservación y de diseño de las infraestructuras

8. Previsión, diseño y gestión de las fuentes de financiación necesarias para la modernización del sistema

Objetivos del Horizonte 2030

1. Disponer de un Sistema de Transportes moderno, en el que la intermodalidad, funcionalidad, capacidad y adecuada conservación sean aspectos fundamentales del mismo

2. Integrar dentro del Sistema de Transportes una red de carreteras de jerarquía racional, con los aeropuertos y los puertos

3. Crear economías de escala en los recintos portuarios, concentrando tráficos que aseguren la rentabilidad de las instalaciones

4. Facilitar la entrada del sector privado en la gestión de las infraestructuras, bajo el liderazgo, rectoría y control del MTOP, sin ceder aspectos relacionados con la planificación estratégica, ordenación y servicios comunes

5. Contribuir en la promoción y facilitación de las actividades de turismo

6. Integrarse en el comercio internacional acorde a las buenas prácticas, recomendaciones y normativa internacionales

7. Potenciar la cohesión y equilibrio del territorio, facilitando la movilidad interna del país, incluida la integración de las regiones especiales

8. Minimizar el impacto en el ambiente del Sistema de Transportes, asegurando la sostenibilidad del mismo en años venideros

9. Alcanzar el punto de autofinanciamiento de las infraestructuras portuarias y aeroportuarias internacionales, a través de un sistema adecuado de tarificación de los servicios, financiando el resto de aeropuertos a través de una política de ayudas a las rutas de servicio público y de los puertos a través de un fondo de compensación Inter portuario

10. Asegurar la estabilidad de la contribución presupuestaria del Estado para la conservación del Sistema de Transportes que esté en gestión directa del Ministerio

Objetivos del horizonte 2037

1. Desarrollo de infraestructuras singulares en cada uno de los modos de transporte y equipamientos logísticos en ubicaciones estratégicas para el desarrollo equilibrado y sostenible del país

2. Completa integración territorial del país, facilitando la accesibilidad de todas las regiones e integrando el Sistema de Transportes en todos los corredores internacionales de tráfico de mercancías

3. Desarrollo del sistema de transportes con visión de potenciar el turismo en aquellas regiones que sean estratégicas en este sentido, dotando al país de un nuevo enfoque y de nuevas herramientas para la atracción de visitantes y de creación de estaciones base estables de los principales operadores de la región

4. Creación de centros logísticos de referencia en la región que permitan atraer tráficos 
de terceros países, convirtiendo a Ecuador, dada su privilegiada ubicación geográfica, en una de las principales plataformas de distribución de determinadas mercancías de la región sudamericana (fruta, pesca, automoción, petróleos, etc.)

5. Constituir un caso de buenas prácticas en la gestión de sistemas de transporte en el ámbito continental. ${ }^{11}$

\section{Plan Estratégico de la Agencia Nacional de Transito - ANT}

La Agencia Nacional de Tránsito, es una entidad adscrita al Ministerio de Transporte y Obras Públicas (MTOP)

De acuerdo con el inciso segundo del artículo 16 de la Ley Orgánica de Transporte Terrestre, Tránsito y Seguridad Vial, es una institución de derecho público con personería jurídica, jurisdicción nacional, presupuesto, patrimonio y régimen administrativo y financiero propio.

Objetivos Estratégicos Institucionales

1. Incrementar la calidad y seguridad del servicio de transporte terrestre y tránsito a nivel nacional.

2. Incrementar la capacidad regulatoria de la ANT para atender los servicios del transporte terrestre, el tránsito y seguridad vial.

3. Incrementar la eficacia de los servicios de la ANT a nivel nacional.

4. Incrementar la eficiencia operacional de la ANT

5. Incrementar el desarrollo del talento humano de la ANT

6. Incrementar el uso eficiente de los recursos de la $\mathrm{ANT}^{12}$

Es necesario resaltar que dichos objetivos están alineados al Plan Nacional del Buen Vivir, Plan del Decenio de Acción para la seguridad Vial, Plan Nacional Estratégico de Movilidad, Plan Estratégico de Transporte y Obras Públicas.

Tabla 1. Proyectos, Planes y Acciones alineadas al Decenio de Acción para la Seguridad Vial $2011-2020$

\begin{tabular}{|l|l}
\hline \multicolumn{1}{|c|}{ Pilar } & \multicolumn{1}{c}{ Proyectos/Planes/Acciones } \\
\hline Gestión de la Seguridad Vial & $\begin{array}{l}\text { *Plan Nacional de Seguridad Vial } \\
\text { *Diseñar metodologías para el desarrollo y } \\
\text { capacitación de seguridad vial }\end{array}$ \\
\hline $\begin{array}{l}\text { Vías de tránsito y movilidad } \\
\text { más seguras }\end{array}$ & $\begin{array}{l}\text { *Plan Nacional de Rutas y Frecuencias } \\
\text { *Centro de Monitoreo Vehicular }\end{array}$ \\
\hline Vehículos más seguros & $\begin{array}{l}\text { *Laboratorio de Homologación Vehicular. } \\
\text { *Plan de Modernización del Transporte } \\
\text { *Sistema Nacional de Revisión Técnica Vehicular. }\end{array}$ \\
\hline Usuarios de vías de tránsito \\
más seguros & $\begin{array}{l}\text { *Proyecto de Seguridad Integral "Transporte } \\
\text { Seguro" } \\
\text { *Generar Modelos de operación para las } \\
\text { modalidades de transporte. }\end{array}$ \\
\hline Respuestas tras los accidentes & $\begin{array}{l}\text { Unidad nacional de coordinación de investigaciones } \\
\text { de accidentes de tránsito }\end{array}$ \\
\hline
\end{tabular}

Fuente: Plan Estratégico de la Agencia Nacional de Tránsito

\footnotetext{
11 Plan Estratégico de Movilidad del MTOP 2013 - 2037

12 Plan Estratégico de Movilidad del MTOP 2013 - 2037
} 


\section{RESULTADOS}

Los accidentes de tránsito de manera general son un gran problema para la sociedad, de manera particular para el Estado desde el punto de vista de la inversión que realiza para evitar aquello o para atender los efectos producidos con políticas de respuesta, también es un serio problema para las personas que directamente están involucradas en el accidente, los efectos son de carácter económico y psicológico principalmente, efectos que replican en la ciudadanía, a menor intensidad pero son parte del diario vivir.

Gran parte de los problemas son generados por el ser humano, y los accidentes de tránsito terrestre no son la excepción, más bien es un caso que permite demostrar con mayor precisión el criterio descrito. Los humanos somos quiénes construimos la infraestructura, fabricamos y damos mantenimiento a los vehículos, establecemos la señalética, realizamos la ley y conducimos los mismos, factores que intervienen directamente en los accidentes de tránsito, por lo que evitar dicho problema depende de la actuación del ser humano en cada uno de sus ámbitos, depende de la gestión que en conjunto podamos realizar, los responsables somos todos, cada quien desde su ámbito con mayor o menor participación, pero responsable al fin.

En el caso de Ecuador, observamos que no se refleja una continuidad e integración adecuada en lo que respecta a los planes de desarrollo respecto de los planes de trabajo institucionales de transporte que nos compete, en principio las modificaciones de los planes se dan en tiempos diferentes, un plan de nivel inferior está alineado a un plan que ya fue removido o cambiado, cuando lo mejor sería que esté alineado al plan superior actual, todos los planes de niveles inferior, deben moverse en función al plan superior que en esta caso es el Plan de Desarrollo Nacional denominado PLAN PARA TODA UNA VIDA. La integración debe ser efectiva, oportuna, congruente, eficaz y trasversal.

La regulación ${ }^{13}$ tardía o en desfase con la planificación macro, no permite obtener los resultados que se aspira para beneficio de los individuos de un país. Vamos a observar ciertos datos que sirven como instrumento para corroborar si las políticas desplegadas, son adecuadas o se requiere reformas y modificaciones. Según los datos obtenidos en las páginas web del Instituto Nacional de Estadísticas y Censos - INEC y la Agencia Nacional de Tránsito - ANT, podemos perfilar la conclusión de que el problema no está resuelto aún, está latente y con tendencia a crecimiento de la tasa de mortalidad causada por los accidentes de tránsito.

\footnotetext{
13 Regulación. - Intervención del Estado en el funcionamiento normal de la actividad económica mediante la emisión de normas con el objeto de vigilar o modificar determinadas condiciones, que pueden ir desde la fijación de los precios de determinados productos, pasando por la defensa de la competencia en el mercado, o de la protección de la situación de los trabajadores. Fuente especificada no válida.
} 
Tabla 2. Fallecidos en Ecuador años 2013 - 2018

\begin{tabular}{|c|c|c|c|c|c|c|c|c|}
\hline \multicolumn{9}{|c|}{ Fallecidos en el Ecuador } \\
\hline PROVINCIAS & 2013 & 2014 & 2015 & 2016 & 2017 & 2018 & Total & Promedio \\
\hline AZUAY & 53 & 85 & 71 & 61 & 92 & 83 & 445 & 127 \\
\hline BOLIVAR & 35 & 32 & 33 & 33 & 24 & 25 & 182 & 52 \\
\hline CAÑAR & 33 & 41 & 61 & 47 & 38 & 48 & 268 & 77 \\
\hline CARCHI & 34 & 15 & 29 & 34 & 17 & 21 & 150 & 43 \\
\hline CHIMBORAZO & 82 & 70 & 82 & 77 & 87 & 52 & 450 & 129 \\
\hline COTOPAXI & 149 & 138 & 113 & 58 & 131 & 79 & 668 & 191 \\
\hline EL ORO & 106 & 95 & 89 & 84 & 86 & 59 & 519 & 148 \\
\hline ESMERALDAS & 60 & 69 & 85 & 48 & 74 & 49 & 385 & 110 \\
\hline GALÀPAGOS & 0 & 1 & 4 & 0 & 0 & 2 & 7 & 2 \\
\hline GUAYAS & 594 & 530 & 477 & 458 & 456 & 374 & 2.889 & 825 \\
\hline IMBABURA & 71 & 74 & 58 & 78 & 81 & 35 & 397 & 113 \\
\hline LOJA & 53 & 57 & 76 & 47 & 25 & 25 & 283 & 81 \\
\hline LOS RIOS & 164 & 141 & 163 & 135 & 180 & 135 & 918 & 262 \\
\hline MANABI & 171 & 134 & 145 & 141 & 150 & 110 & 851 & 243 \\
\hline $\begin{array}{c}\text { MORONA } \\
\text { SANTIAGO }\end{array}$ & 28 & 34 & 26 & 36 & 31 & 23 & 178 & 51 \\
\hline NAPO & 23 & 24 & 27 & 13 & 25 & 13 & 125 & 36 \\
\hline ORELLANA & 43 & 56 & 25 & 20 & 15 & 24 & 183 & 52 \\
\hline PASTAZA & 16 & 23 & 14 & 11 & 10 & 13 & 87 & 25 \\
\hline PICHINCHA & 301 & 448 & 327 & 331 & 375 & 269 & 2.051 & 586 \\
\hline SANTA ELENA & 37 & 34 & 32 & 43 & 40 & 29 & 215 & 61 \\
\hline $\begin{array}{c}\text { SANTO } \\
\text { DOMINGO DE } \\
\text { LOS SACHILAS } \\
\end{array}$ & 110 & 83 & 75 & 68 & 72 & 69 & 477 & 136 \\
\hline SUCUMBİOS & 21 & 29 & 24 & 40 & 35 & 18 & 167 & 48 \\
\hline TUNGURAHUA & 68 & 91 & 90 & 93 & 92 & 64 & 498 & 142 \\
\hline $\begin{array}{c}\text { ZAMORA } \\
\text { CHIMCHIPE }\end{array}$ & 25 & 18 & 12 & 11 & 17 & 8 & 91 & 26 \\
\hline Total & 4.290 & 4.336 & 4.153 & 3.983 & 4.170 & 3.645 & 12.484 & 3.567 \\
\hline Promedio & 95 & 97 & 89 & 82 & 90 & 68 & 520 & \\
\hline Promedio trimestre & 23,72 & 24,19 & 22,27 & 20,49 & 22,43 & 22,60 & & \\
\hline
\end{tabular}

Fuente: https://www.ant.gob.ec/index.php/noticias/estadisticas\#.V8oG1PnhDIU

En el cuadro 1 se observa que las provincias que tienen mayor número de fallecidos son la provincia del Guayas con 2.889 fallecidos entre enero del año 2013 hasta septiembre del año 2018, seguido de la provincia de Pichincha con 2.051 fallecidos en el mismo período. Estas dos provincias son justamente las que mayor número de habitantes tienen en su territorio, lo cual explica los primeros lugares en el número de fallecidos por causa de los accidentes de tránsito, sin embargo, una medición más efectiva y acertada, es realizando una relación entre el número de fallecidos y el número de habitantes, lo cual nos arroja los siguientes resultados. 
Figura 1. Fallecidos Ecuador

Fallecidos por cada 100.000 habitantes en Ecuador - año 2013

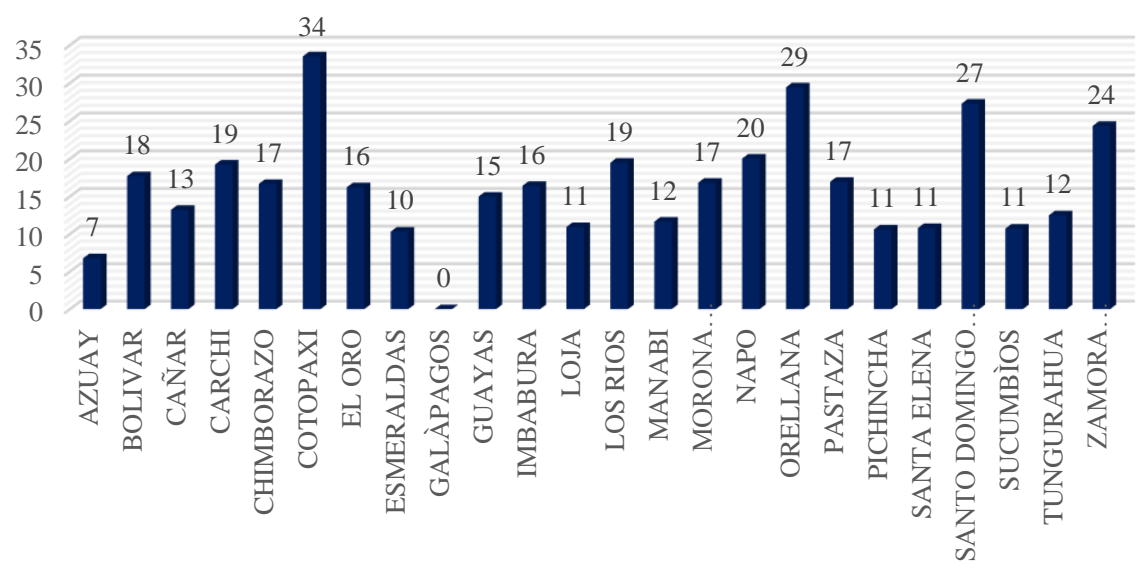

Fuente: https://www.ant.gob.ec/index.php/noticias/estadisticas\#.V8oG1PnhDIU

Para el año 2013, las tres provincias que tienen la tasa más alta de mortalidad, son respectivamente la provincia de Cotopaxi con $34 \%$, Orellana con el $29 \%$ y la provincia de Santo Domingo de los Sachilas con el 27\%, mientras que Galápagos, Azuay y Esmeraldas son las provincias que tienen menor tasa de mortalidad.

Figura 2. Fallecidos en el Ecuador 2014

Fallecidos por cada 100.000 habitantes en Ecuador - año 2014

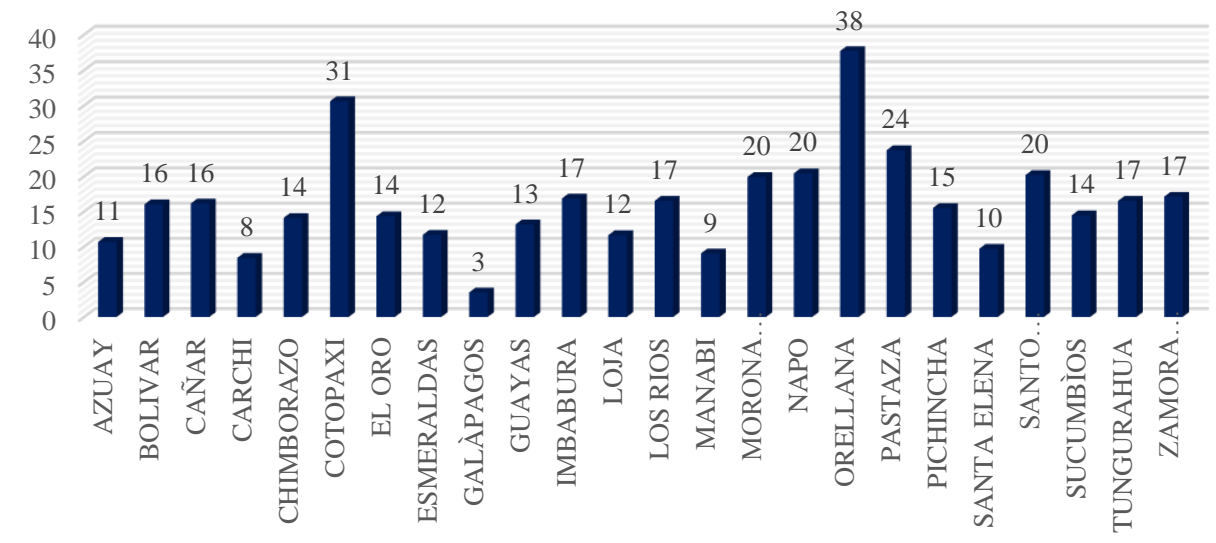

Fuente: https://www.ant.gob.ec/index.php/noticias/estadisticas\#.V8oG1PnhDIU

En el año 2014, es la provincia de Orellana la que obtiene la tasa más alta, con el 38\%, la provincia de Cotopaxi que tenía el primer lugar en el año 2013, ahora se ubica en el segundo lugar con una tasa del $31 \%$ y la provincia de Pastaza sube al tercer lugar con una tasa del 24\%, esto significa que por cada 100.000, en el año 2014, fallecieron 24 personas. 
Figura 3. Fallecidos en el Ecuador 2015

Fallecidos por cada 100.000 habitantes en Ecuador - año 2015

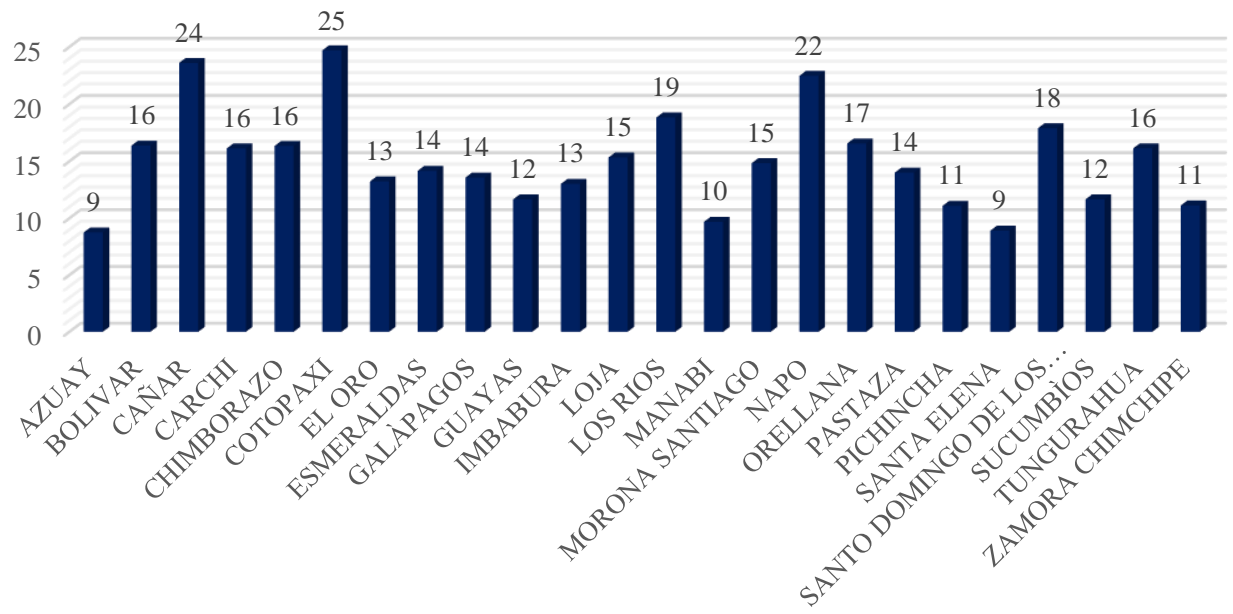

Fuente: https://www.ant.gob.ec/index.php/noticias/estadisticas\#.V8oG1PnhDIU

La provincia de Cotopaxi retoma el primer lugar que tenía en el 2013, si bien baja de $34 \%$ a 25\%, es la tasa más alta para este año. En segundo lugar, está la provincia de Cañar con un $24 \%$ y la provincia de Napo es la tercera en la clasificación de las provincias que mayores muertes tienen por temas de accidentes de tránsito.

Figura 4. Fallecidos en el Ecuador 2016

Fallecidos por cada 100.000 habitantes en Ecuador - año 2016

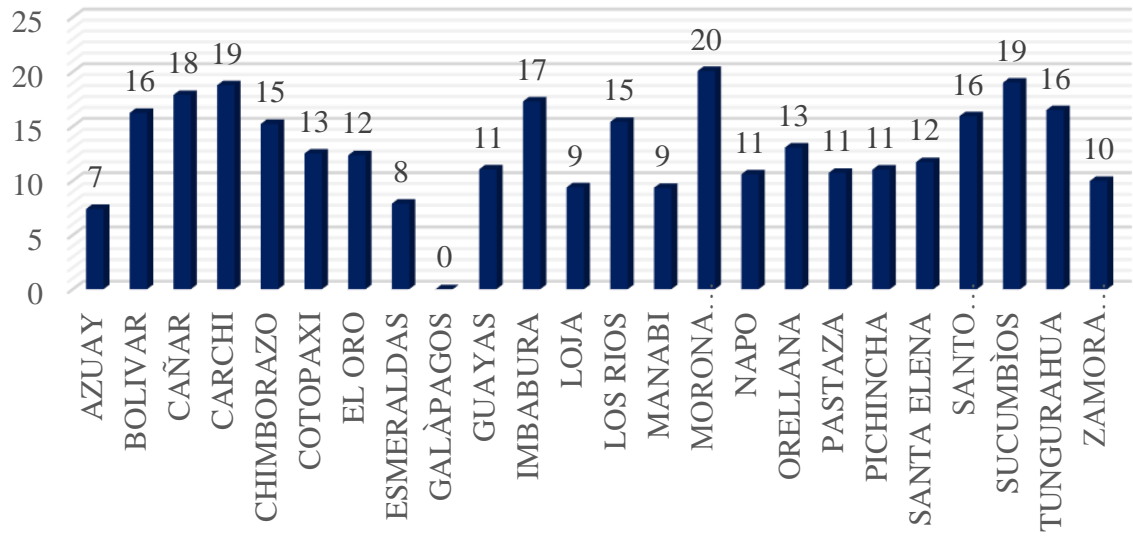

Fuente: $\underline{\text { https://www.ant.gob.ec/index.php/noticias/estadisticas\#.V8oG1PnhDIU }}$

En el año 2016, la provincia de Morona tiene una tasa del 20\% ubicándose en el primer lugar, de manera general podemos indicar que la tasa en promedio bajo, pero no es suficiente para resolver al problema. En segundo lugar, tenemos las provincias de Sucumbíos y Carchi. Orellana la que obtiene la tasa más alta, siendo ésta del $38 \%$, la provincia de Cotopaxi que tenía el primer lugar en el año 2013, ahora se ubica en el segundo lugar con una tasa del $31 \%$ y la provincia de Pastaza sube al tercer lugar con una tasa del 24\%, esto significa que por cada 100.000, en el año 2014, fallecieron 24 personas. 
Figura 5. Fallecidos en el Ecuador 2017

Fallecidos por cada 100.000 habitantes en Ecuador - año 2017

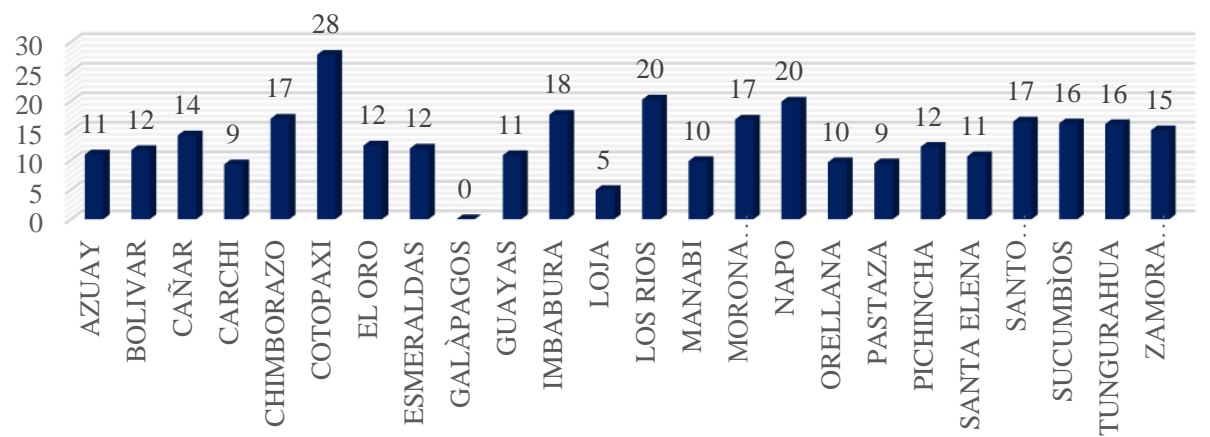

Fuente: $\underline{\text { https://www.ant.gob.ec/index.php/noticias/estadisticas\#.V8oG1PnhDIU }}$

En el año 2014, es la provincia de Orellana la que obtiene la tasa más alta, siendo ésta del $38 \%$, la provincia de Cotopaxi que tenía el primer lugar en el año 2013, ahora se ubica en el segundo lugar con una tasa del $31 \%$ y la provincia de Pastaza sube al tercer lugar con una tasa del 24\%, esto significa que por cada 100.000, en el año 2014, fallecieron 24 personas.

Figura 5. Fallecidos en el Ecuador 2018

Fallecidos por cada 100.000 habitantes en Ecuador (promedio por año - 2013 a septiembre 2018)

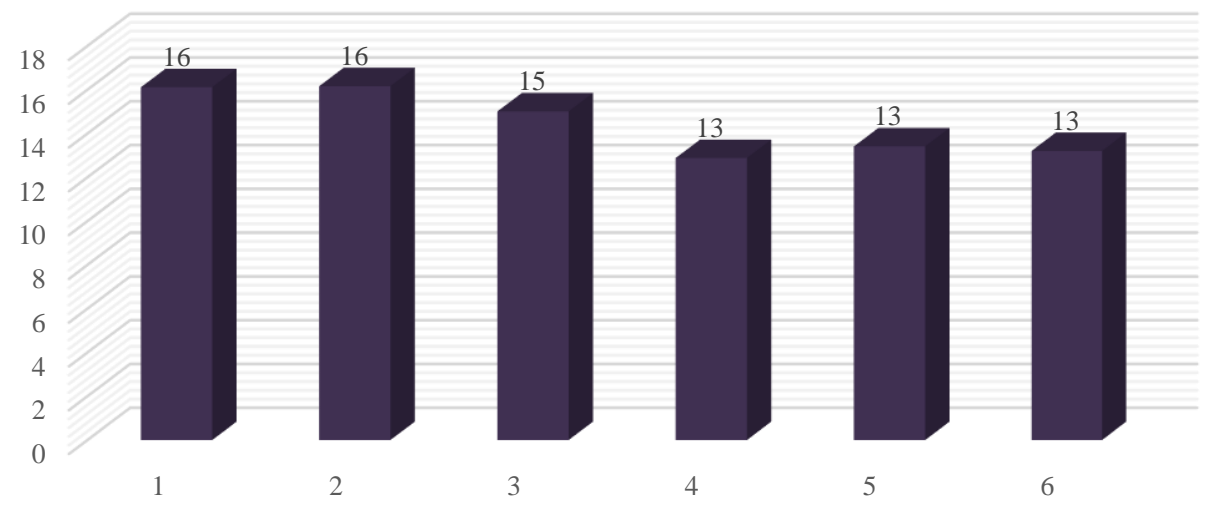

Fuente: https://www.ant.gob.ec/index.php/noticias/estadisticas\#.V8oG1PnhDIU

\section{CONCLUCIONES}

- Los trabajos que realizan los organismos nacionales e internacionales demuestran que existe un alto grado de interés por dar solución a tan delicada problemática en beneficio de la humanidad.

- Parte de la solución a la problemática está en el campo político, lamentablemente pensamos de manera individual, sectorial y no en los de un país más habitable para todos. 
- Los datos obtenidos de la Agencia Nacional de Tránsito demuestran que el problema persiste, es más serio en ciertas provincias con relación a otras.

- Se puede establecer, que el endurecimiento de las sanciones para contravenciones de tránsito ha tenido efecto positivo, al reducirse la cantidad de siniestros; es decir las políticas regulatorias en este sentido están cumpliendo con su objetivo.

- La normativa vigente, si ha permitido o aportado a que esta tasa baje a otros niveles, no obstante, su aplicación en mayor número de infractores permitirá mejor resultados.

\section{RECOMENDACIONES}

Antes

- Alinearse a las organizaciones internacionales y observar los trabajos y recomendaciones planteadas.

- Integrar a las instituciones e involucrados a fin de trabajar en una solución de patrocinada por el Estado de manera integrada.

- Promover o generar una cultura vial que se acerque a los estándares positivos internacionales.

- Levantar una plataforma de información para acompañar a las metas, indicadores, estratégicas, objetivos y en si a la planificación total.

- Generar proyectos que respondan y atiendan a los sujetos involucrados en los accidentes, incluido los fallecidos y familiares.

Durante

- Dar soporte y apoyo a las familias de los involucrados en siniestros, fallecidos y lesionados.

- Obtener información que sirva de base para trabajar sobre la prevención en el mediano, corto y largo plazo

- Cumplir con lo planificado.

Después

- Realizar estudios que sirvan de base para la mitigación del problema.

- Tener retroalimentación constante para dar respuestas inmediatas.

\section{Bibliografía}

INEC. (diciembre de 2016). Proyección de la Población del Ecuador basada en el Censo de Población y vivienda 2010. Obtenido de www. ecuadorencifras.gob.ec.

INEC. (diciembre de 2016). Vehículos matriculados año 2016. Obtenido de www.ecuadorencifras.gob.ec.

ANT, DEP, QUITO. (agosto de 2018). www.ecuadorencifras.gob.ec.

COIP - Muñoz, Nicolás R. (octubre de 2014). nicolasrmunoz.com/infracciones-detransito-en-el-ecuador-con-el-nuevo-coip/.

Metro Ecuador. (marzo de 2018). Obtenido de https://www.metroecuador.com.ec/ec/noticias/2018/03/09/6-infracciones-detransito-en-las-que-se-pierde-puntos-en-la-licencia.html. 
Valles, J. (2013). Tráfico y Transporte. USA: McGraw-Hill.

Asamblea Nacional Constituyente. (2014). Ley Orgánica de Transporte Terrestre

Transito y Seguridad Vial. Obtenido de https://www.turismo.gob.ec/wpcontent/uploads/2016/04/LEY-ORGANICA-DE-TRANSPORTE-

TERRESTRE-TRANSITO-Y-SEGURIDAD-VIAL.pdf

Sapag, N., \& Chain, R. (2008). Preparación y Evaluación de Proyectos. Bogotá: McGraw Hill.

Córdoba, M. (2011). Formulación y Evaluación de Proyectos. Bogotá: Eco Ediciones.

Serna G., H. (1997). Gerencia estratégica: planeación y gestión - teoría y metodología. Bogotá: 3R Editores.

Morales C., J. A., \& Morales C., A. (2014). Planeación financiera. México: Grupo Editorial Patria.

M. P., \& E. L. (2016). Microeconomía, Versión para Latinoamérica. México: Apolo, S.A.

Arthur Andersen, S. A. (1999). DICCIONARIO DE ECONOMÍA Y NEGOCIOS. MADRID: ESPASA.

Murcia, J. M., \& Piraquive, F. D. (2014). Proyectos, formulación y criterios de evaluación. Bogotá: Alfaomega. 


\section{PARA CITAR EL ARTÍCULO INDEXADO}

Chamba Bastidas, L., Moreno Albuja, M., Cortez Manzano, R., \& Carrera Silva, K. (2019). Necesidad de generar proyectos alineados a la política regulatoria del sector transporté, para combatir la tasa de mortalidad promovida por los accidentes de tránsito y que afecta a la economía del Ecuador. Visionario Digital, 3(3), 157-178. https://doi.org/10.33262/visionariodigital.v3i3.667

\section{Ciencia}

El artículo que se publica es de exclusiva responsabilidad de los autores y no necesariamente reflejan el pensamiento de la Revista Visionario Digital.

El artículo queda en propiedad de la revista y, por tanto, su publicación parcial y/o total en otro medio tiene que ser autorizado por el director de la Revista Visionario Digital.
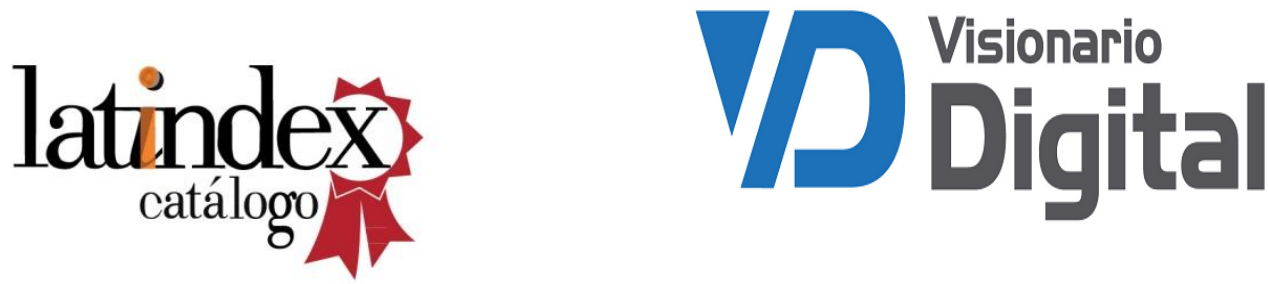